\title{
Chasing telomeres, not red herrings, in evolutionary ecology
}

Heredity (2011) 107, 372-373; doi:10.1038/hdy.2011.14; published online 16 March 2011

Like a burning fuse, telomere shortening can trigger cellular senescence and is implicated in accelerated ageing (von Zglinicki, 2002). Consequently, the role of telomeres is a hot topic in studies of longevity and lifehistory traits (Monaghan, 2010). In their recent critical review, Horn et al. (2010) caution that this enthusiasm has led to a lack of rigour, particularly in the application of a quantitative real-time PCR (qPCR) technique to measure relative telomere length (RTL) (Cawthon, 2002). We agree that researchers eager to leap into telomere biology should fully comprehend the potential methodological pitfalls of RTL. Horn et al. overstate the problems, however, and give the mistaken impression that these are insurmountable. Unfortunately, their critique only further muddies the waters. Here, we seek to rectify some of their misconceptions. We also highlight the existing resources available to maximise resolution in studies using qPCR to estimate RTLs and provide positive guidelines for their implementation.

The first criticism by Horn et al. of RTL concerns intraand inter-assay variation in PCR amplification or reaction efficiency, which refers to how much of the target DNA sequence is amplified in each cycle. Their concern is that '... most studies do not check the efficiencies of their samples, and it is simply assumed that there is a higher chance of consistency between standard and sample efficiency if the standard is optimized to a value around $100 \%$ (the theoretical perfect amplification)'. They argue this is a 'dangerous assumption' especially because DNA samples may be stored with a variety of PCR inhibitors. Variation in amplification efficiency is a source of measurement error that reduces precision in estimated RTLs, but Horn et al.'s critique implies this variation will create a systematic bias-leading to the false identification of significant effects (that is, statistical type I errors). Such biases could only occur if the variation in reaction efficiencies (due to differences in sample storage, storage buffers or DNA extraction techniques) is systematically correlated with treatments or explanatory factors. Otherwise, it simply generates unbiased (albeit unwanted) noise in estimates of RTL. As with any method, increasing precision instills greater confidence in estimates of effect sizes (negative or positive). Contradictory to the impression given by Horn et al., reaction efficiency has for many years been recognised as critical to the precision of qPCR (Pfaffl, 2001; Smith et al., 2002). The gene-quantification website, for example, lists over 30 papers from the last 5 years that address this issue (http:/ / www.gene-quantification.info). Recent studies describe methods to best estimate sample efficiencies and show how their use greatly reduces the coefficient of variation $(\mathrm{CV})$ in estimates of RTL using qPCR, as does the incorporation of a multiplex design (see Supplementary data).
The second concern raised by Horn et al. is the selection of an appropriate reference or 'single copy' gene, which is needed to standardise the telomere PCR product against total DNA concentration. They worry that reference genes used for RTL sometimes "possess similar pseudo-genes across the genome, yet this is seldom considered'. Again, Horn et al. miss the point that a reference gene for an RTL assay need not be single copy, but rather non-variable in copy number among samples used in the study. The usual label of 'single copy

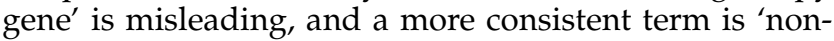
variable copy number' (non-VCN) gene. As yet, there is no searchable database of non-VCNs and developing them for each new target species is a time-consuming and problematic step in the RTL approach. Nevertheless, by adopting a systematic approach to non-VCN development, this hurdle can be readily overcome (see Supplementary data).

Third, Horn et al. doubt the potential for quantifying RTL values allowing comparison among studies. There are two basic approaches to achieve this aim: the first is to use an external sample of known telomere concentration as a standard, and the second approach is by using the so-called 'gold standard' telomere restriction fragment (TRF) method (or other methods) to 'calibrate' a set of samples. Neither approach is assumption free. Horn et al. rightly point out that reaction efficiencies of external 'highly purified commercial samples' will differ compared with regular samples that likely include co-extracted PCR inhibitors. Yet, this does not, in itself, disqualify them as standards because (as explained above) a constant difference in efficiency cannot cause a systematic error biasing only a subset of samples. What is important is to estimate efficiencies as accurately as possible and incorporate them in the analysis of qPCR reactions. Efficiencies can be estimated separately for the telomere and non-VCN gene reactions in the assay for both the controls and samples using external analysis software (see Supplementary data).

Estimates of RTL can also be quantified by regression against absolute measurements. Indeed, because of the relative nature of RTLs, only the control requires quantification (for example, a sample with an RTL of 1.5 has an absolute mean telomere length that is 1.5 times that of the control). Caution is needed, however, when using TRF to calibrate RTL values because the two methods measure slightly different aspects of telomeres. The TRF method gives a distribution of lengths of the telomeric sequence, from which a mean is often taken, whereas the RTL method estimates the total amount of the telomeric sequence per genome relative to a control. Birds, for instance, often have interstitial telomeric repeats and ultralong telomeres $(>100 \mathrm{~kb})$. Neither is measured using TRF, whereas they are included in the RTL method. Thus, conversion of RTLs to absolute values based on TRF validation is not sufficient to allow 
meta-analysis of TL dynamics among species or higher taxa.

Finally, Horn et al. downplay the advantages of RTL estimation. The requirement of only small starting DNA amounts together with the potential for rapid, high-throughput analysis offers many advantages over traditional approaches to telomere length estimation. Many of the problems discussed here and elsewhere (Aviv, 2009; Horn et al., 2010) have been addressed in other fields of qPCR research (for example, MIQE guidelines; Bustin et al., 2009). We applaud efforts to 'raise the bar' on methodology, but efforts should be focussed on addressing actual problems rather than red herrings. Therefore, we outline specific steps that can be followed to improve the reliability of RTL estimation (see Supplementary data). The use of RTL estimation holds great promise for the study of telomere dynamics, and, given its benefits, we encourage the widespread adoption of RTL as a tool in molecular ecology and evolution.

\section{Conflict of interest}

The authors declare no conflict of interest.

S Smith ${ }^{1,2}$, C Turbill ${ }^{1}$ and DJ Penn ${ }^{2}$

${ }^{1}$ Research Institute of Wildlife Ecology,

Department of Integrative Biology and Evolution,

University of Veterinary Medicine Vienna, Vienna, Austria and
${ }^{2}$ Konrad Lorenz Institute of Ethology, Department of Integrative Biology and Evolution, University of

Veterinary Medicine Vienna,

Vienna, Austria

E-mail: steve.smith@fiwi.at

\section{References}

Aviv A (2009). Commentary: Raising the bar on telomere epidemiology. Int J Epidemiol 38: 1735-1736.

Bustin SA, Benes V, Garson JA, Hellemans J, Huggett J, Kubista M et al. (2009). The MIQE guidelines: minimum information for publication of quantitative real-time PCR experiments. Clin Chem 55: 611-622.

Cawthon RM (2002). Telomere measurement by quantitative PCR. Nucl Acids Res 30: e47.

Horn T, Robertson BC, Gemmell NJ (2010). The use of telomere length in ecology and evolutionary biology. Heredity 105: 497-506.

Monaghan P (2010). Crossing the great divide: telomeres and ecology. Heredity 105: 574-575.

Pfaffl MW (2001). A new mathematical model for relative quantification in real-time RT-PCR. Nucl Acids Res 29: e45.

Smith S, Vigilant L, Morin PA (2002). The effects of sequence length and oligonucleotide mismatches on $5^{\prime}$ exonuclease assay efficiency-art. no. e111. Nucl Acids Res 30: E111.

von Zglinicki T (2002). Oxidative stress shortens telomeres. Trends Biochem Sci 27: 339-344.

Supplementary Information accompanies the paper on Heredity website (http://www.nature.com/hdy) 